\title{
Existence of solutions for a general quasilinear elliptic system via perturbation method
}

Yujuan Jiao ${ }^{1 *}$, Shengmao $\mathrm{Fu}^{2}$ and Yanli Wang ${ }^{3}$

\section{"Correspondence:} jsjyj@xbmu.edu.cn

${ }^{1}$ College of Mathematics and Computer Science, Northwest University for Nationalities, Lanzhou, 730124, P.R. China

Full list of author information is available at the end of the article

\section{Abstract}

In this paper, we consider the following quasilinear elliptic system:

$$
\begin{cases}-\sum_{i, j=1}^{N} D_{j}\left(a_{i j}(x, u) D_{i} u\right)+\frac{1}{2} \sum_{i, j=1}^{N} D_{s} a_{i j}(x, u) D_{i} u D_{j} u=\frac{2 \alpha}{\alpha+\beta}|u|^{\alpha-2}|v|^{\beta} u, & x \in \Omega, \\ -\sum_{i, j=1}^{N} D_{j}\left(b_{i j}(x, v) D_{i} v\right)+\frac{1}{2} \sum_{i, j=1}^{N} D_{s} b_{i j}(x, v) D_{i} v D_{j} v=\frac{2 \beta}{\alpha+\beta}|u|^{\alpha}|v|^{\beta-2} v, & x \in \Omega, \\ u=0, \quad v=0, & x \in \partial \Omega,\end{cases}
$$

where $D_{i} u=\frac{\partial u}{\partial x_{i}}, D_{s} a_{i j}(x, u)=\frac{\partial}{\partial u} a_{i j}(x, u), D_{s} b_{i j}(x, v)=\frac{\partial}{\partial v} b_{i j}(x, v), \alpha>2, \beta>2$, $\alpha+\beta<2 \cdot 2^{*}, 2^{*}=\frac{2 N}{N-2}$ is the critical Sobolev exponent and $\Omega \subset \mathbb{R}^{N}(N \geq 3)$ is a bounded smooth domain. By using the perturbation method, we establish the existence of both positive and negative solutions for this system.

MSC: $35 \mathrm{~J} 60 ; 35 \mathrm{~B} 33$

Keywords: quasilinear elliptic system; positive solution; negative solution; perturbation method

\section{Introduction}

Let us consider the following quasilinear elliptic system:

$$
\begin{cases}-\sum_{i, j=1}^{N} D_{j}\left(a_{i j}(x, u) D_{i} u\right)+\frac{1}{2} \sum_{i, j=1}^{N} D_{s} a_{i j}(x, u) D_{i} u D_{j} u=\frac{2 \alpha}{\alpha+\beta}|u|^{\alpha-2}|v|^{\beta} u, & x \in \Omega, \\ -\sum_{i, j=1}^{N} D_{j}\left(b_{i j}(x, v) D_{i} v\right)+\frac{1}{2} \sum_{i, j=1}^{N} D_{s} b_{i j}(x, v) D_{i} v D_{j} v=\frac{2 \beta}{\alpha+\beta}|u|^{\alpha}|v|^{\beta-2} v, & x \in \Omega, \\ u=0, \quad v=0, & x \in \partial \Omega,\end{cases}
$$

where $D_{i} u=\frac{\partial u}{\partial x_{i}}, D_{s} a_{i j}(x, u)=\frac{\partial}{\partial u} a_{i j}(x, u), D_{s} b_{i j}(x, v)=\frac{\partial}{\partial v} b_{i j}(x, v), \alpha>2, \beta>2, \alpha+\beta<2$. $2^{*}, 2^{*}=\frac{2 N}{N-2}$ is the critical Sobolev exponent and $\Omega \subset \mathbb{R}^{N}(N \geq 3)$ is a bounded smooth domain. This system includes the following special class of system with $a_{i j}(x, u)=(1+$ $\left.u^{2}\right) \delta_{i j}, b_{i j}(x, v)=\left(1+v^{2}\right) \delta_{i j}$, i.e.,

$$
\begin{cases}-\Delta u-\frac{1}{2} u \Delta\left(u^{2}\right)=\frac{2 \alpha}{\alpha+\beta}|u|^{\alpha-2}|v|^{\beta} u, & x \in \Omega, \\ -\Delta v-\frac{1}{2} v \triangle\left(v^{2}\right)=\frac{2 \beta}{\alpha+\beta}|u|^{\alpha}|v|^{\beta-2} v, & x \in \Omega, \\ u=0, \quad v=0, & x \in \partial \Omega,\end{cases}
$$

which is referred to as the so-called modified nonlinear Schrödinger system.

\section{黛 Springer}

๑ 2013 Jiao et al.; licensee Springer. This is an Open Access article distributed under the terms of the Creative Commons Attribution License (http://creativecommons.org/licenses/by/2.0), which permits unrestricted use, distribution, and reproduction in any medium, provided the original work is properly cited. 
Our assumptions on the functions $a_{i j}$ and $b_{i j}$ are as follows.

$\left(\mathrm{A}_{1}\right)$ The functions $a_{i j} \in C^{1}(\bar{\Omega} \times \mathbb{R}, \mathbb{R}), b_{i j} \in C^{1}(\bar{\Omega} \times \mathbb{R}, \mathbb{R}), a_{i j}=a_{j i}, b_{i j}=b_{j i}, i, j=1,2, \ldots, N$.

$\left(\mathrm{A}_{2}\right)$ There exist constants $a_{0}, a_{1}, b_{0}, b_{1}$ satisfying $a_{1} \geq a_{0}>0, b_{1} \geq b_{0}>0,(\alpha+\beta-2) a_{0}>$ $2 a_{1}$ and $(\alpha+\beta-2) b_{0}>2 b_{1}$ such that

$$
\begin{aligned}
& a_{0}\left(1+s^{2}\right)|\xi|^{2} \leq \sum_{i, j=1}^{N} a_{i j}(x, s) \xi_{i} \xi_{j} \leq a_{1}\left(1+s^{2}\right)|\xi|^{2} \\
& b_{0}\left(1+s^{2}\right)|\xi|^{2} \leq \sum_{i, j=1}^{N} b_{i j}(x, s) \xi_{i} \xi_{j} \leq b_{1}\left(1+s^{2}\right)|\xi|^{2}
\end{aligned}
$$

for $x \in \bar{\Omega}, \xi \in \mathbb{R}^{N}, s \in \mathbb{R}$

$\left(\mathrm{A}_{3}\right)$

$$
\begin{aligned}
& 0 \leq \sum_{i, j=1}^{N} D_{s} a_{i j}(x, s) s \xi_{i} \xi_{j} \leq 2 \sum_{i, j=1}^{N} a_{i j}(x, s) \xi_{i} \xi_{j}, \\
& 0 \leq \sum_{i, j=1}^{N} D_{s} b_{i j}(x, s) s \xi_{i} \xi_{j} \leq 2 \sum_{i, j=1}^{N} b_{i j}(x, s) \xi_{i} \xi_{j}
\end{aligned}
$$

for $x \in \bar{\Omega}, \xi \in \mathbb{R}^{N}, s \in \mathbb{R}$

In recent years, much attention has been devoted to the quasilinear Schrödinger equation of the following form:

$$
-\triangle u+\lambda V(x) u-k \triangle\left(u^{2}\right) u=|u|^{p-2} u, \quad x \in \mathbb{R}^{N} .
$$

See, for example, [1] where Poppenberg et al. proved the existence of a positive ground state solution by using a constrained minimization argument. Using a change of variables, Liu et al. [2] used an Orlicz space to prove the existence of a soliton solution for equation (1.2) via the mountain pass theorem. Colin and Jeanjean [3] also made use of a change of variables but worked in the Sobolev space $H^{1}\left(\mathbb{R}^{N}\right)$. They proved the existence of a positive solution for equation (1.2) from the classical results given by Berestycki and Lions [4]. Liu et al. [5] established the existence of both one-sign and nodal ground states of soliton-type solutions for equation (1.2) by the Nehari method. By using the Nehari manifold method and the concentration compactness principle (see [6]) in the Orlicz space, Guo and Tang [7] considered the following quasilinear Schrödinger system:

$$
\begin{cases}-\Delta u+(\lambda a(x)+1) u-\frac{1}{2}\left(\Delta|u|^{2}\right) u=\frac{2 \alpha}{\alpha+\beta}|u|^{\alpha-2}|v|^{\beta} u, & x \in \mathbb{R}^{N}, \\ -\Delta u+(\lambda b(x)+1) u-\frac{1}{2}\left(\Delta|u|^{2}\right) u=\frac{2 \beta}{\alpha+\beta}|u|^{\alpha}|\nu|^{\beta-2} v, & x \in \mathbb{R}^{N}, \\ u(x) \rightarrow 0, \quad v(x) \rightarrow 0, & |x| \rightarrow \infty,\end{cases}
$$

with $a(x) \geq 0, b(x) \geq 0$ having a potential well and $\alpha>2, \beta>2, \alpha+\beta<2 \cdot 2^{*}$, and they proved the existence of a ground state solution for system (1.3) which localizes near the potential well int $a^{-1}(0)$ for $\lambda$ large enough. Guo and Tang [8] considered also ground state solutions of the single quasilinear Schrödinger equation corresponding to system (1.3) by 
the same methods and obtained similar results. In particular, by the perturbation method, Liu et al. [9] considered the existence and multiplicity of solutions for the following quasilinear equation of the form

$$
\begin{cases}\sum_{i, j=1}^{N} D_{j}\left(a_{i j}(x, u) D_{i} u\right)-\frac{1}{2} \sum_{i, j=1}^{N} D_{s} a_{i j}(x, u) D_{i} u D_{j} u+f(x, u)=0, & x \in \Omega, \\ u=0, & x \in \partial \Omega\end{cases}
$$

under suitable assumptions.

It is worth pointing out that the existence of one-bump or multi-bump bound state solutions for the related semilinear Schrödinger equation (1.2) for $k=0$ has been extensively studied. One can see Bartsch and Wang [10], Ambrosetti et al. [11], Ambrosetti et al. [12], Byeon and Wang [13], Cingolani and Lazzo [14], Cingolani and Nolasco [15], Del Pino and Felmer [16, 17], Floer and Weinstein [18], Oh [19, 20] and the references therein.

Motivated by the single equation (1.4), the purpose of this paper is to study the existence of both positive and negative solutions for the coupled quasilinear system (1.1). We mainly follow the idea of Liu et al. [9] to perturb the functional and obtain our main results. We point out that the procedure to system (1.1) is not trivial at all. Since the appearance of the quasilinear terms $\sum_{i, j=1}^{N} D_{j}\left(a_{i j}(x, u) D_{i} u\right)-\frac{1}{2} \sum_{i, j=1}^{N} D_{s} a_{i j}(x, u) D_{i} u D_{j} u$ and $\sum_{i, j=1}^{N} D_{j}\left(b_{i j}(x, v) D_{i} v\right)-\frac{1}{2} \sum_{i, j=1}^{N} D_{s} b_{i j}(x, v) D_{i} v D_{j} v$, we need more delicate estimates.

The paper is organized as follows. In Section 2, we introduce a perturbation of the functional and give our main results (Theorem 2.1 and Theorem 2.2). In Section 3, we verify the Palais-Smale condition for the perturbed functional. Section 4 is devoted to some asymptotic behavior of the sequences $\left\{\left(u_{n}, v_{n}\right)\right\} \subset W_{0}^{1,4}(\Omega) \times W_{0}^{1,4}(\Omega)$ and $\left\{\mu_{n}\right\} \subset(0,1]$ satisfying some conditions. Finally, our main results will be proved in Section 5 .

Throughout this paper, we will use the same $C$ to denote various generic positive constants, and we will use $o(1)$ to denote quantities that tend to 0 .

\section{Perturbation of the functional and main results}

In order to obtain the desired existence of solutions for system (1.1), in this section, we introduce a perturbation of the functional and give our main results.

The weak form of system (1.1) is

$$
\begin{gathered}
\int_{\Omega} \sum_{i, j=1}^{N} a_{i j}(x, u) D_{i} u D_{j} \varphi+\frac{1}{2} \int_{\Omega} \sum_{i, j=1}^{N} D_{s} a_{i j}(x, u) D_{i} u D_{j} u \varphi \\
+\int_{\Omega} \sum_{i, j=1}^{N} b_{i j}(x, v) D_{i} v D_{j} \psi+\frac{1}{2} \int_{\Omega} \sum_{i, j=1}^{N} D_{s} b_{i j}(x, v) D_{i} v D_{j} v \psi \\
-\frac{2 \alpha}{\alpha+\beta} \int_{\Omega}|u|^{\alpha-2}|v|^{\beta} u \varphi-\frac{2 \beta}{\alpha+\beta} \int_{\Omega}|u|^{\alpha}|\nu|^{\beta-2} \nu \psi=0
\end{gathered}
$$

for all $(\varphi, \psi) \in C_{0}^{\infty}(\Omega) \times C_{0}^{\infty}(\Omega)$, which is formally the variational formulation of the following functional:

$$
I_{0}(u, v)=\frac{1}{2} \int_{\Omega} \sum_{i, j=1}^{N} a_{i j}(x, u) D_{i} u D_{j} u+\frac{1}{2} \int_{\Omega} \sum_{i, j=1}^{N} b_{i j}(x, v) D_{i} v D_{j} v-\frac{2}{\alpha+\beta} \int_{\Omega}|u|^{\alpha}|v|^{\beta} .
$$


We may define the derivative of $I_{0}$ at $(u, v)$ in the direction of $(\varphi, \psi) \in C_{0}^{\infty}(\Omega) \times C_{0}^{\infty}(\Omega)$ as follows:

$$
\begin{aligned}
\left\langle I_{0}^{\prime}(u, v),(\varphi, \psi)\right\rangle= & \int_{\Omega} \sum_{i, j=1}^{N} a_{i j}(x, u) D_{i} u D_{j} \varphi+\frac{1}{2} \int_{\Omega} \sum_{i, j=1}^{N} D_{s} a_{i j}(x, u) D_{i} u D_{j} u \varphi \\
& +\int_{\Omega} \sum_{i, j=1}^{N} b_{i j}(x, v) D_{i} v D_{j} \psi+\frac{1}{2} \int_{\Omega} \sum_{i, j=1}^{N} D_{s} b_{i j}(x, v) D_{i} v D_{j} v \psi \\
& -\frac{2 \alpha}{\alpha+\beta} \int_{\Omega}|u|^{\alpha-2}|\nu|^{\beta} u \varphi-\frac{2 \beta}{\alpha+\beta} \int_{\Omega}|u|^{\alpha}|v|^{\beta-2} \nu \psi
\end{aligned}
$$

We call $(u, v)$ a critical point of $I_{0}$ if $(u, v) \in W_{0}^{1,2}(\Omega) \times W_{0}^{1,2}(\Omega), \int_{\Omega} u^{2}|\nabla u|^{2}<\infty$, $\int_{\Omega} v^{2}|\nabla v|^{2}<\infty$ and $\left\langle I_{0}^{\prime}(u, v),(\varphi, \psi)\right\rangle=0$ for all $(\varphi, \psi) \in C_{0}^{\infty}(\Omega) \times C_{0}^{\infty}(\Omega)$. That is, $(u, v)$ is a weak solution for system (1.1).

When we consider system (1.1) by using the classical critical point theory, we encounter the difficulties due to the lack of an appropriate working space. In general, it seems that there is no suitable space in which the variational functional $I_{0}$ possesses both smoothness and compactness properties. For smoothness, one would need to work in a space smaller than $W_{0}^{1,2}(\Omega)$ to control the term involving the quasilinear term in system (1.1), but it seems impossible to obtain bounds for $(P S)_{c}$ sequence in this setting. Several ideas and approaches, such as minimizations [1,21], the Nehari method [5] and change of variables $[2,3]$, have been used in recent years to overcome the difficulties. In this paper, we consider the perturbed functional

$$
\begin{aligned}
I_{\mu}(u, v)= & \frac{1}{4} \mu \int_{\Omega}\left(|\nabla u|^{4}+|\nabla v|^{4}\right)+I_{0}(u, v) \\
= & \frac{1}{4} \mu \int_{\Omega}\left(|\nabla u|^{4}+|\nabla v|^{4}\right)+\frac{1}{2} \int_{\Omega} \sum_{i, j=1}^{N} a_{i j}(x, u) D_{i} u D_{j} u \\
& +\frac{1}{2} \int_{\Omega} \sum_{i, j=1}^{N} b_{i j}(x, v) D_{i} v D_{j} v-\frac{2}{\alpha+\beta} \int_{\Omega}|u|^{\alpha}|v|^{\beta},
\end{aligned}
$$

where $\mu \in(0,1]$ is a parameter. Then it is easy to see that $I_{\mu}$ is a $C^{1}$-functional on $W_{0}^{1,4}(\Omega) \times$ $W_{0}^{1,4}(\Omega)$. We can define also the derivative of $I_{\mu}$ at $(u, v)$ in the direction of $(\varphi, \psi)$ as follows:

$$
\begin{aligned}
\left\langle I_{\mu}^{\prime}(u, v),(\varphi, \psi)\right\rangle= & \mu \int_{\Omega}|\nabla u|^{2} \nabla u \nabla \varphi+\mu \int_{\Omega}|\nabla v|^{2} \nabla v \nabla \psi \\
& +\int_{\Omega} \sum_{i, j=1}^{N} a_{i j}(x, u) D_{i} u D_{j} \varphi+\frac{1}{2} \int_{\Omega} \sum_{i, j=1}^{N} D_{s} a_{i j}(x, u) D_{i} u D_{j} u \varphi \\
& +\int_{\Omega} \sum_{i, j=1}^{N} b_{i j}(x, v) D_{i} v D_{j} \psi+\frac{1}{2} \int_{\Omega} \sum_{i, j=1}^{N} D_{s} b_{i j}(x, v) D_{i} v D_{j} v \psi \\
& -\frac{2 \alpha}{\alpha+\beta} \int_{\Omega}|u|^{\alpha-2}|v|^{\beta} u \varphi-\frac{2 \beta}{\alpha+\beta} \int_{\Omega}|u|^{\alpha}|v|^{\beta-2} v \psi
\end{aligned}
$$


for all $(\varphi, \psi) \in C_{0}^{\infty}(\Omega) \times C_{0}^{\infty}(\Omega)$. The idea of this paper is to obtain the existence of the critical points of $I_{\mu}$ for $\mu>0$ small and establish suitable estimates for the critical points as $\mu \rightarrow 0$ so that we may pass to the limit to get the solutions for the original system (1.1).

Our main results are as follows.

Theorem 2.1 Assume that $\left(\mathrm{A}_{1}\right)-\left(\mathrm{A}_{3}\right)$ hold, $\alpha>2, \beta>2$ and $\alpha+\beta<2 \cdot 2^{*}$. Let $\mu_{n} \rightarrow 0$ and let $\left\{\left(u_{n}, v_{n}\right)\right\} \subset W_{0}^{1,4}(\Omega) \times W_{0}^{1,4}(\Omega)$ be a sequence of critical points of $I_{\mu_{n}}$ satisfying $I_{\mu_{n}}^{\prime}\left(u_{n}, v_{n}\right)=0$ and $I_{\mu_{n}}\left(u_{n}, v_{n}\right) \leq C$ for some $C$ independent of $n$. Then, up to a subsequence,

$$
\begin{aligned}
& u_{n} \rightarrow u, \quad v_{n} \rightarrow v \quad \text { in } W_{0}^{1,2}(\Omega), \\
& u_{n} \nabla u_{n} \rightarrow u \nabla u, \quad v_{n} \nabla v_{n} \rightarrow v \nabla v \quad \text { in } L^{2}(\Omega), \\
& \mu_{n} \int_{\Omega}\left(\left|\nabla u_{n}\right|^{4}+\left|\nabla v_{n}\right|^{4}\right) \rightarrow 0, \\
& I_{\mu_{n}}^{\prime}\left(u_{n}, v_{n}\right) \rightarrow I_{0}^{\prime}(u, v)
\end{aligned}
$$

as $n \rightarrow \infty$, and $(u, v)$ is a critical point of $I_{0}$.

Theorem 2.2 Assume that $\left(\mathrm{A}_{1}\right)-\left(\mathrm{A}_{3}\right)$ hold, $\alpha>2, \beta>2$ and $\alpha+\beta<2 \cdot 2^{*}$. Then $I_{\mu}$ has a positive critical point $\left(u_{\mu}, v_{\mu}\right)$ and a negative critical point $\left(\widetilde{u}_{\mu}, \widetilde{v}_{\mu}\right)$, and $\left(u_{\mu}, v_{\mu}\right)\left(\right.$ resp., $\left.\left(\widetilde{u}_{\mu}, \widetilde{v}_{\mu}\right)\right)$ converges to a positive (resp., negative) solution for system (1.1) as $\mu \rightarrow 0$.

Notation We denote by $\|\cdot\|$ the norm of $W_{0}^{1,4}(\Omega)$ and by $|\cdot|_{s}$ the norm of $L^{s}(\Omega)(1 \leq s<$ $+\infty)$.

\section{Compactness of the perturbed functional}

In this section, we verify the Palais-Smale condition $\left((P S)_{c}\right.$ condition in short) for the perturbed functional $I_{\mu}(u, v)$. We have the following proposition.

Proposition 3.1 For $\mu>0$ fixed, the functional $I_{\mu}(u, v)$ satisfies $(P S)_{c}$ condition for all $c \in \mathbb{R}$. That is, any sequence $\left\{\left(u_{n}, v_{n}\right)\right\} \subset W_{0}^{1,4}(\Omega) \times W_{0}^{1,4}(\Omega)$ satisfying, for $c \in \mathbb{R}$,

$$
\begin{aligned}
& I_{\mu}\left(u_{n}, v_{n}\right) \rightarrow c, \\
& I_{\mu}^{\prime}\left(u_{n}, v_{n}\right) \rightarrow 0 \quad \text { strongly in }\left(W_{0}^{1,4}(\Omega) \times W_{0}^{1,4}(\Omega)\right)^{*}
\end{aligned}
$$

has a strongly convergent subsequence in $W_{0}^{1,4}(\Omega) \times W_{0}^{1,4}(\Omega)$, where $\left(W_{0}^{1,4}(\Omega) \times W_{0}^{1,4}(\Omega)\right)^{*}$ is the dual space of $W_{0}^{1,4}(\Omega) \times W_{0}^{1,4}(\Omega)$.

To give the proof of Proposition 3.1, we need the following lemma firstly.

Lemma 3.2 Suppose that a sequence $\left\{\left(u_{n}, v_{n}\right)\right\} \subset W_{0}^{1,4}(\Omega) \times W_{0}^{1,4}(\Omega)$ satisfies (3.1) and (3.2). Then

$$
\limsup _{n \rightarrow \infty}\left\|\left(u_{n}, v_{n}\right)\right\|^{4} \leq\left(\frac{1}{4}-\frac{1}{\alpha+\beta}\right)^{-1} \mu^{-1} c .
$$


Proof It follows from (3.1) and (3.2) that

$$
\begin{aligned}
c+ & o(1)-\frac{1}{\alpha+\beta} o(1)\left\|\left(u_{n}, v_{n}\right)\right\| \\
= & I_{\mu}\left(u_{n}, v_{n}\right)-\frac{1}{\alpha+\beta}\left\langle I_{\mu}^{\prime}\left(u_{n}, v_{n}\right),\left(u_{n}, v_{n}\right)\right\rangle \\
= & \left(\frac{1}{4}-\frac{1}{\alpha+\beta}\right) \mu \int_{\Omega}\left(\left|\nabla u_{n}\right|^{4}+\left|\nabla v_{n}\right|^{4}\right) \\
& +\left(\frac{1}{2}-\frac{1}{\alpha+\beta}\right) \int_{\Omega} \sum_{i, j=1}^{N} a_{i j}(x, u) D_{i} u D_{j} u+\left(\frac{1}{2}-\frac{1}{\alpha+\beta}\right) \int_{\Omega} \sum_{i, j=1}^{N} b_{i j}(x, v) D_{i} v D_{j} v \\
& -\frac{1}{2(\alpha+\beta)} \int_{\Omega} \sum_{i, j=1}^{N} D_{s} a_{i j}(x, u) D_{i} u D_{j} u-\frac{1}{2(\alpha+\beta)} \int_{\Omega} \sum_{i, j=1}^{N} D_{s} b_{i j}(x, v) D_{i} v D_{j} v \\
\geq & \left(\frac{1}{4}-\frac{1}{\alpha+\beta}\right) \mu \int_{\Omega}\left(|\nabla u|^{4}+|\nabla v|^{4}\right)+\frac{(\alpha+\beta-2) a_{0}-2 a_{1}}{2(\alpha+\beta)} \int_{\Omega}\left(1+u_{n}^{2}\right)\left|\nabla u_{n}\right|^{2} \\
& +\frac{(\alpha+\beta-2) b_{0}-2 b_{1}}{2(\alpha+\beta)}\left(1+v_{n}^{2}\right)\left|\nabla v_{n}\right|^{2} \\
\geq & \left(\frac{1}{4}-\frac{1}{\alpha+\beta}\right) \mu \int_{\Omega}\left(|\nabla u|^{4}+|\nabla v|^{4}\right) .
\end{aligned}
$$

Thus we have

$$
\limsup _{n \rightarrow \infty}\left\|\left(u_{n}, v_{n}\right)\right\|^{4} \leq\left(\frac{1}{4}-\frac{1}{\alpha+\beta}\right)^{-1} \mu^{-1} c .
$$

This completes the proof of Lemma 3.2.

Now we give the proof of Proposition 3.1.

Proof of Proposition 3.1 From Lemma 3.2, we know that $\left\{\left(u_{n}, v_{n}\right)\right\}$ is bounded in $W_{0}^{1,4}(\Omega) \times$ $W_{0}^{1,4}(\Omega)$. So there exists a subsequence of $\left\{\left(u_{n}, v_{n}\right)\right\}$, still denoted by $\left\{\left(u_{n}, v_{n}\right)\right\}$, such that

$$
\begin{aligned}
& \left(u_{n}, v_{n}\right) \rightarrow(u, v) \quad \text { weakly in } W_{0}^{1,4}(\Omega) \times W_{0}^{1,4}(\Omega) \text { as } n \rightarrow \infty, \\
& u_{n} \rightarrow u, \quad v_{n} \rightarrow v \quad \text { strongly in } L^{s}(\Omega) \text { as } n \rightarrow \infty \text { for any } 2<s<2 \cdot 2^{*} .
\end{aligned}
$$

Now we prove that $\left(u_{n}, v_{n}\right) \rightarrow(u, v)$ in $W_{0}^{1,4}(\Omega) \times W_{0}^{1,4}(\Omega)$. In $(2.5)$, choosing $(\varphi, \psi)=\left(u_{n}-\right.$ $\left.u_{m}, v_{n}-v_{m}\right)$, we have

$$
\begin{aligned}
o(1) & \left\|\left(u_{n}-u_{m}, v_{n}-v_{m}\right)\right\| \\
= & \left\langle I_{\mu}^{\prime}\left(u_{n}, v_{n}\right)-I_{\mu}^{\prime}\left(u_{m}, v_{m}\right),\left(u_{n}-u_{m}, v_{n}-v_{m}\right)\right\rangle \\
= & \mu \int_{\Omega}\left(\left|\nabla u_{n}\right|^{2} \nabla u_{n}-\left|\nabla u_{m}\right|^{2} \nabla u_{m}\right)\left(\nabla u_{n}-\nabla u_{m}\right) \\
& +\mu \int_{\Omega}\left(\left|\nabla v_{n}\right|^{2} \nabla v_{n}-\left|\nabla v_{m}\right|^{2} \nabla v_{m}\right)\left(\nabla v_{n}-\nabla v_{m}\right) \\
& +\int_{\Omega} \sum_{i, j=1}^{N}\left(a_{i j}\left(x, u_{n}\right) D_{i} u_{n}-a_{i j}\left(x, u_{m}\right) D_{i} u_{m}\right)\left(D_{j} u_{n}-D_{j} u_{m}\right)
\end{aligned}
$$




$$
\begin{aligned}
& +\int_{\Omega} \sum_{i, j=1}^{N}\left(b_{i j}\left(x, v_{n}\right) D_{i} v_{n}-b_{i j}\left(x, v_{m}\right) D_{i} v_{m}\right)\left(D_{j} v_{n}-D_{j} v_{m}\right) \\
& +\frac{1}{2} \int_{\Omega} \sum_{i, j=1}^{N}\left(D_{s} a_{i j}\left(x, u_{n}\right) D_{i} u_{n} D_{j} u_{n}-D_{s} a_{i j}\left(x, u_{m}\right) D_{i} u_{m} D_{j} u_{m}\right)\left(u_{n}-u_{m}\right) \\
& +\frac{1}{2} \int_{\Omega} \sum_{i, j=1}^{N}\left(D_{s} b_{i j}\left(x, v_{n}\right) D_{i} v_{n} D_{j} v_{n}-D_{s} b_{i j}\left(x, v_{m}\right) D_{i} v_{m} D_{j} v_{m}\right)\left(v_{n}-v_{m}\right) \\
& -\frac{2 \alpha}{\alpha+\beta} \int_{\Omega}\left(\left|u_{n}\right|^{\alpha-2}\left|v_{n}\right|^{\beta} u_{n}-\left|u_{m}\right|^{\alpha-2}\left|v_{m}\right|^{\beta} u_{m}\right)\left(u_{n}-u_{m}\right) \\
& -\frac{2 \beta}{\alpha+\beta} \int_{\Omega}\left(\left|u_{n}\right|^{\alpha}\left|v_{n}\right|^{\beta-2} v_{n}-\left|u_{m}\right|^{\alpha}\left|v_{m}\right|^{\beta-2} v_{m}\right)\left(v_{n}-v_{m}\right) .
\end{aligned}
$$

We may estimate the terms involved as follows:

$$
\begin{aligned}
& \mu \int_{\Omega}\left(\left|\nabla u_{n}\right|^{2} \nabla u_{n}-\left|\nabla u_{m}\right|^{2} \nabla u_{m}\right)\left(\nabla u_{n}-\nabla u_{m}\right) \geq \frac{1}{4} \mu \int_{\Omega}\left|\nabla u_{n}-\nabla u_{m}\right|^{4}, \\
& \mu \int_{\Omega}\left(\left|\nabla v_{n}\right|^{2} \nabla v_{n}-\left|\nabla v_{m}\right|^{2} \nabla v_{m}\right)\left(\nabla v_{n}-\nabla v_{m}\right) \geq \frac{1}{4} \mu \int_{\Omega}\left|\nabla v_{n}-\nabla v_{m}\right|^{4}, \\
& \int_{\Omega} \sum_{i, j=1}^{N}\left(a_{i j}\left(x, u_{n}\right) D_{i} u_{n}-a_{i j}\left(x, u_{m}\right) D_{i} u_{m}\right)\left(D_{j} u_{n}-D_{j} u_{m}\right) \\
& =\int_{\Omega} \sum_{i, j=1}^{N} a_{i j}\left(x, u_{n}\right) D_{i}\left(u_{n}-u_{m}\right) D_{j}\left(u_{n}-u_{m}\right) \\
& +\int_{\Omega} \sum_{i, j=1}^{N}\left(a_{i j}\left(x, u_{n}\right)-a_{i j}\left(x, u_{m}\right)\right) D_{i} u_{m} D_{j}\left(u_{n}-u_{m}\right) \\
& \geq \int_{\Omega} \sum_{i, j=1}^{N} D_{s} a_{i j}\left(x, t u_{n}+(1-t) u_{m}\right)\left(u_{n}-u_{m}\right) D_{i} u_{m} D_{j}\left(u_{n}-u_{m}\right) \\
& \geq-C\left|u_{n}-u_{m}\right|_{4}\left\|u_{m}\right\|\left(\left\|u_{n}\right\|+\left\|u_{m}\right\|\right) \\
& \rightarrow 0 \text { as } m, n \rightarrow \infty \text { for some } t \in(0,1) \text {, } \\
& \int_{\Omega} \sum_{i, j=1}^{N}\left(b_{i j}\left(x, v_{n}\right) D_{i} v_{n}-b_{i j}\left(x, v_{m}\right) D_{i} v_{m}\right)\left(D_{j} v_{n}-D_{j} v_{m}\right) \\
& =\int_{\Omega} \sum_{i, j=1}^{N} b_{i j}\left(x, v_{n}\right) D_{i}\left(v_{n}-v_{m}\right) D_{j}\left(v_{n}-v_{m}\right) \\
& +\int_{\Omega} \sum_{i, j=1}^{N}\left(b_{i j}\left(x, v_{n}\right)-b_{i j}\left(x, v_{m}\right)\right) D_{i} v_{m} D_{j}\left(v_{n}-v_{m}\right) \\
& \geq \int_{\Omega} \sum_{i, j=1}^{N} D_{s} b_{i j}\left(x, \tau v_{n}+(1-\tau) v_{m}\right)\left(v_{n}-v_{m}\right) D_{i} v_{m} D_{j}\left(v_{n}-v_{m}\right) \\
& \geq-C\left|v_{n}-v_{m}\right|_{4}\left\|v_{m}\right\|\left(\left\|v_{n}\right\|+\left\|v_{m}\right\|\right) \\
& \rightarrow 0 \quad \text { as } m, n \rightarrow \infty \text { for some } \tau \in(0,1) \text {, }
\end{aligned}
$$




$$
\begin{aligned}
& \frac{1}{2}\left|\int_{\Omega} \sum_{i, j=1}^{N}\left(D_{s} a_{i j}\left(x, u_{n}\right) D_{i} u_{n} D_{j} u_{n}-D_{s} a_{i j}\left(x, u_{m}\right) D_{i} u_{m} D_{j} u_{m}\right)\left(u_{n}-u_{m}\right)\right| \\
& \leq \frac{1}{2} \int_{\Omega} \sum_{i, j=1}^{N}\left|D_{s} a_{i j}\left(x, u_{n}\right) D_{i} u_{n} D_{j} u_{n}\left(u_{n}-u_{m}\right)\right| \\
& +\frac{1}{2} \int_{\Omega} \sum_{i, j=1}^{N}\left|D_{s} a_{i j}\left(x, u_{n}\right) D_{i} u_{n} D_{j} u_{n}\left(u_{n}-u_{m}\right)\right| \\
& \leq C\left(\left\|u_{n}\right\|^{2}+\left\|u_{m}\right\|^{2}\right)\left|u_{n}-u_{m}\right|_{4} \\
& \rightarrow 0 \text { as } m, n \rightarrow \infty \\
& \frac{1}{2}\left|\int_{\Omega} \sum_{i, j=1}^{N}\left(D_{s} b_{i j}\left(x, v_{n}\right) D_{i} v_{n} D_{j} v_{n}-D_{s} b_{i j}\left(x, v_{m}\right) D_{i} v_{m} D_{j} v_{m}\right)\left(v_{n}-v_{m}\right)\right| \\
& \leq \frac{1}{2} \int_{\Omega} \sum_{i, j=1}^{N}\left|D_{s} b_{i j}\left(x, v_{n}\right) D_{i} v_{n} D_{j} v_{n}\left(v_{n}-v_{m}\right)\right| \\
& +\frac{1}{2} \int_{\Omega} \sum_{i, j=1}^{N}\left|D_{s} b_{i j}\left(x, v_{n}\right) D_{i} v_{n} D_{j} v_{n}\left(v_{n}-v_{m}\right)\right| \\
& \leq C\left(\left\|v_{n}\right\|^{2}+\left\|v_{m}\right\|^{2}\right)\left|v_{n}-v_{m}\right|_{4} \\
& \rightarrow 0 \quad \text { as } m, n \rightarrow \infty \\
& \frac{2 \alpha}{\alpha+\beta}\left|\int_{\Omega}\left(\left|u_{n}\right|^{\alpha-2}\left|v_{n}\right|^{\beta} u_{n}-\left|u_{m}\right|^{\alpha-2}\left|v_{m}\right|^{\beta} u_{m}\right)\left(u_{n}-u_{m}\right)\right| \\
& \leq \frac{2 \alpha}{\alpha+\beta} \int_{\Omega}\left(\left|u_{n}\right|^{\alpha-1}\left|v_{n}\right|^{\beta}+\left|u_{m}\right|^{\alpha-1}\left|v_{m}\right|^{\beta}\right)\left|u_{n}-u_{m}\right| \\
& \leq \frac{2 \alpha}{\alpha+\beta}\left(\left|u_{n}\right|_{\alpha+\beta}^{\alpha-1}\left|v_{n}\right|_{\alpha+\beta}^{\beta}+\left|u_{m}\right|_{\alpha+\beta}^{\alpha-1}\left|v_{m}\right|_{\alpha+\beta}^{\beta}\right)\left|u_{n}-u_{m}\right|_{\alpha+\beta} \\
& \rightarrow 0 \quad \text { as } m, n \rightarrow \infty \\
& \frac{2 \beta}{\alpha+\beta}\left|\int_{\Omega}\left(\left|u_{n}\right|^{\alpha}\left|v_{n}\right|^{\beta-2} v_{n}-\left|u_{m}\right|^{\alpha}\left|v_{m}\right|^{\beta-2} v_{m}\right)\left(v_{n}-v_{m}\right)\right| \\
& \leq \frac{2 \beta}{\alpha+\beta} \int_{\Omega}\left(\left|u_{n}\right|^{\alpha}\left|v_{n}\right|^{\beta-1}+\left|u_{m}\right|^{\alpha}\left|v_{m}\right|^{\beta-1}\right)\left|v_{n}-v_{m}\right| \\
& \leq \frac{2 \beta}{\alpha+\beta}\left(\left|u_{n}\right|_{\alpha+\beta}^{\alpha}\left|v_{n}\right|_{\alpha+\beta}^{\beta-1}+\left|u_{m}\right|_{\alpha+\beta}^{\alpha}\left|v_{m}\right|_{\alpha+\beta}^{\beta-1}\right)\left|v_{n}-v_{m}\right|_{\alpha+\beta} \\
& \rightarrow 0 \quad \text { as } m, n \rightarrow \infty \text {. }
\end{aligned}
$$

Returning to (3.3), we have

$$
\frac{1}{4} \mu \int_{\Omega}\left(\left|\nabla u_{n}-\nabla u_{m}\right|^{4}+\left|\nabla v_{n}-\nabla v_{m}\right|^{4}\right) \leq o(1)\left\|\left(u_{n}-u_{m}, v_{n}-v_{m}\right)\right\|+o(1)
$$

which implies that $\left\|\left(u_{n}-u_{m}, v_{n}-v_{m}\right)\right\| \rightarrow 0$, i.e., $\left(u_{n}, u_{m}\right) \rightarrow(u, v)$ in $W_{0}^{1,4}(\Omega) \times W_{0}^{1,4}(\Omega)$. This completes the proof of Proposition 3.1. 


\section{Some asymptotic behavior}

Proposition 3.1 enables us to apply minimax argument to the functional $I_{\mu}(u, v)$. In this section, we also study the behavior of the sequences $\left\{\left(u_{n}, v_{n}\right)\right\} \subset W_{0}^{1,4}(\Omega) \times W_{0}^{1,4}(\Omega)$ and $\left\{\mu_{n}\right\} \subset(0,1]$ satisfying

$$
\begin{aligned}
& \mu_{n} \rightarrow 0, \\
& I_{\mu_{n}}\left(u_{n}, v_{n}\right) \rightarrow c, \\
& \left\|I_{\mu_{n}}^{\prime}\left(u_{n}, v_{n}\right)\right\|^{*} \rightarrow 0 .
\end{aligned}
$$

The following proposition is the key of this section.

Proposition 4.1 Assume that the sequences $\left\{\left(u_{n}, v_{n}\right)\right\} \subset W_{0}^{1,4}(\Omega) \times W_{0}^{1,4}(\Omega)$ and $\left\{\mu_{n}\right\} \subset$ $(0,1]$ satisfy (4.1)-(4.3). Then, after extracting a sequence, still denoted by $n$, we have

$$
\begin{aligned}
& \left(u_{n}, v_{n}\right) \rightarrow(u, v) \quad \text { in } W_{0}^{1,2}(\Omega) \times W_{0}^{1,2}(\Omega), \\
& \left(u_{n} \nabla u_{n}, v_{n} \nabla v_{n}\right) \rightarrow(u \nabla u, v \nabla v) \quad \text { in } L^{2}(\Omega) \times L^{2}(\Omega)
\end{aligned}
$$

and

$$
\left(u_{n}(x), v_{n}(x)\right) \rightarrow(u(x), v(x)) \quad \text { a.e. } x \in \Omega
$$

as $n \rightarrow \infty$.

Proof Similar to the proof of Lemma 3.2, by (4.1)-(4.3), we have

$$
\begin{aligned}
C \geq & I_{\mu_{n}}\left(u_{n}, v_{n}\right)-\frac{1}{\alpha+\beta}\left\langle I_{\mu_{n}}^{\prime}\left(u_{n}, v_{n}\right),\left(u_{n}, v_{n}\right)\right\rangle \\
\geq & \left(\frac{1}{4}-\frac{1}{\alpha+\beta}\right) \mu_{n} \int_{\Omega}\left(\left|\nabla u_{n}\right|^{4}+\left|\nabla v_{n}\right|^{4}\right) \\
& +\frac{(\alpha+\beta-2) a_{0}-2 a_{1}}{2(\alpha+\beta)} \int_{\Omega}\left(1+u_{n}^{2}\right)\left|\nabla u_{n}\right|^{2} \\
& +\frac{(\alpha+\beta-2) b_{0}-2 b_{1}}{2(\alpha+\beta)} \int_{\Omega}\left(1+v_{n}^{2}\right)\left|\nabla v_{n}\right|^{2} .
\end{aligned}
$$

Thus

$$
\mu_{n} \int_{\Omega}\left(\left|\nabla u_{n}\right|^{4}+\left|\nabla v_{n}\right|^{4}\right)+\int_{\Omega}\left(\left|\nabla u_{n}\right|^{2}+\left|\nabla v_{n}\right|^{2}\right)+\int_{\Omega}\left(u_{n}^{2}\left|\nabla u_{n}\right|^{2}+v_{n}^{2}\left|\nabla v_{n}\right|^{2}\right) \leq C
$$

for some $C$ independent of $n$. Then, up to a subsequence, we have

$$
\begin{aligned}
& \left(u_{n}, v_{n}\right) \rightarrow(u, v) \quad \text { in } W_{0}^{1,2}(\Omega) \times W_{0}^{1,2}(\Omega), \\
& \left(u_{n} \nabla u_{n}, v_{n} \nabla v_{n}\right) \rightarrow(u \nabla u, v \nabla v) \quad \text { in } L^{2}(\Omega) \times L^{2}(\Omega)
\end{aligned}
$$

and

$$
\left(u_{n}(x), v_{n}(x)\right) \rightarrow(u(x), v(x)) \quad \text { a.e. } x \in \Omega
$$

as $n \rightarrow \infty$. This completes the proof of Proposition 4.1. 


\section{Proof of main results}

In this section, we give the proof of our main results. Firstly, we prove Theorem 2.1.

Proof of Theorem 2.1 Note that $\left(u_{n}, v_{n}\right)$ satisfies the following equation:

$$
\begin{aligned}
& \mu_{n} \int_{\Omega}\left|\nabla u_{n}\right|^{2} \nabla u_{n} \nabla \varphi+\mu_{n} \int_{\Omega}\left|\nabla v_{n}\right|^{2} \nabla v_{n} \nabla \psi \\
& +\int_{\Omega} \sum_{i, j=1}^{N} a_{i j}\left(x, u_{n}\right) D_{i} u_{n} D_{j} \varphi+\frac{1}{2} \int_{\Omega} \sum_{i, j=1}^{N} D_{s} a_{i j}\left(x, u_{n}\right) D_{i} u_{n} D_{j} u_{n} \varphi \\
& \quad+\int_{\Omega} \sum_{i, j=1}^{N} b_{i j}\left(x, v_{n}\right) D_{i} v_{n} D_{j} \psi+\frac{1}{2} \int_{\Omega} \sum_{i, j=1}^{N} D_{s} b_{i j}\left(x, v_{n}\right) D_{i} v_{n} D_{j} v_{n} \psi \\
& \quad-\frac{2 \alpha}{\alpha+\beta} \int_{\Omega}\left|u_{n}\right|^{\alpha-2}\left|v_{n}\right|^{\beta} u_{n} \varphi-\frac{2 \beta}{\alpha+\beta} \int_{\Omega}\left|u_{n}\right|^{\alpha}\left|v_{n}\right|^{\beta-2} v_{n} \psi=0
\end{aligned}
$$

for all $(\varphi, \psi) \in W_{0}^{1,4}(\Omega) \times W_{0}^{1,4}(\Omega)$. Since

$$
\left(\int_{\Omega}\left|u_{n}\right|^{\frac{4 N}{N-2}}\right)^{\frac{N-2}{N}} \leq C \int_{\Omega} \sum_{i, j=1}^{N} a_{i j}\left(x, u_{n}\right) D_{i} u_{n} D_{j} u_{n} \leq C
$$

and

$$
\left(\int_{\Omega}\left|v_{n}\right|^{\frac{4 N}{N-2}}\right)^{\frac{N-2}{N}} \leq C \int_{\Omega} \sum_{i, j=1}^{N} b_{i j}\left(x, v_{n}\right) D_{i} v_{n} D_{j} v_{n} \leq C .
$$

By Moser's iteration, we have

$$
\left|u_{n}\right|_{L^{\infty}} \leq C, \quad\left|v_{n}\right|_{L^{\infty}} \leq C
$$

Hence

$$
|u|_{L^{\infty}} \leq C, \quad|v|_{L^{\infty}} \leq C
$$

for some $C$ independent of $n$. To show that $(u, v)$ is a critical point of $I_{0}$, we use some arguments in $[22,23]$ (see more references therein). In (5.1), we choose $\varphi=\xi \exp \left(-M u_{n}\right)$, $\psi=\eta \exp \left(-M v_{n}\right)$, where $\xi \in C_{0}^{\infty}(\Omega), \xi \geq 0, \eta \in C_{0}^{\infty}(\Omega), \eta \geq 0$ and $M>0$ is a constant. Substituting $(\varphi, \psi)$ into (5.1), we have

$$
\begin{aligned}
0= & \mu_{n} \int_{\Omega}\left|\nabla u_{n}\right|^{2} \nabla u_{n}\left(\nabla \xi \exp \left(-M u_{n}\right)-\xi \nabla u_{n} \exp \left(-M u_{n}\right)\right) \\
& +\mu_{n} \int_{\Omega}\left|\nabla v_{n}\right|^{2} \nabla v_{n}\left(\nabla \eta \exp \left(-M v_{n}\right)-\eta \nabla v_{n} \exp \left(-M v_{n}\right)\right) \\
& +\int_{\Omega} \sum_{i, j=1}^{N} a_{i j}\left(x, u_{n}\right) D_{i} u_{n}\left(D_{j} \xi \exp \left(-M u_{n}\right)-M \xi D_{j} u_{n} \exp \left(-M u_{n}\right)\right) \\
& +\int_{\Omega} \sum_{i, j=1}^{N} b_{i j}\left(x, v_{n}\right) D_{i} v_{n}\left(D_{j} \eta \exp \left(-M v_{n}\right)-M \eta D_{j} v_{n} \exp \left(-M v_{n}\right)\right)
\end{aligned}
$$




$$
\begin{aligned}
& +\frac{1}{2} \int_{\Omega} \sum_{i, j=1}^{N} D_{s} a_{i j}\left(x, u_{n}\right) D_{i} u_{n} D_{j} u_{n} \xi \exp \left(-M u_{n}\right) \\
& +\frac{1}{2} \int_{\Omega} \sum_{i, j=1}^{N} D_{s} b_{i j}\left(x, v_{n}\right) D_{i} v_{n} D_{j} v_{n} \eta \exp \left(-M v_{n}\right) \\
& -\frac{2 \alpha}{\alpha+\beta} \int_{\Omega}\left|u_{n}\right|^{\alpha-2}\left|v_{n}\right|^{\beta} u_{n} \xi \exp \left(-M u_{n}\right)-\frac{2 \beta}{\alpha+\beta} \int_{\Omega}\left|u_{n}\right|^{\alpha}\left|v_{n}\right|^{\beta-2} v_{n} \eta \exp \left(-M v_{n}\right) \\
& \leq \mu_{n} \int_{\Omega}\left|\nabla u_{n}\right|^{2} \nabla u_{n} \nabla \xi \exp \left(-M u_{n}\right)+\mu_{n} \int_{\Omega}\left|\nabla v_{n}\right|^{2} \nabla v_{n} \nabla \eta \exp \left(-M v_{n}\right) \\
& +\int_{\Omega} \sum_{i, j=1}^{N} a_{i j}\left(x, u_{n}\right) D_{i} u_{n} D_{j} \xi \exp \left(-M u_{n}\right)+\int_{\Omega} \sum_{i, j=1}^{N} b_{i j}\left(x, v_{n}\right) D_{i} v_{n} D_{j} \eta \exp \left(-M v_{n}\right) \\
& -\int_{\Omega} \sum_{i, j=1}^{N}\left(M a_{i j}\left(x, u_{n}\right)-\frac{1}{2} D_{s} a_{i j}\left(x, u_{n}\right)\right) D_{i} u_{n} D_{j} u_{n} \xi \exp \left(-M u_{n}\right) \\
& -\int_{\Omega} \sum_{i, j=1}^{N}\left(M b_{i j}\left(x, v_{n}\right)-\frac{1}{2} D_{s} b_{i j}\left(x, v_{n}\right)\right) D_{i} v_{n} D_{j} v_{n} \eta \exp \left(-M v_{n}\right) \\
& -\frac{2 \alpha}{\alpha+\beta} \int_{\Omega}\left|u_{n}\right|^{\alpha-2}\left|v_{n}\right|^{\beta} u_{n} \xi \exp \left(-M u_{n}\right) \\
& \quad-\frac{2 \beta}{\alpha+\beta} \int_{\Omega}\left|u_{n}\right|^{\alpha}\left|v_{n}\right|^{\beta-2} v_{n} \eta \exp \left(-M v_{n}\right) .
\end{aligned}
$$

Note that $M a_{i j}\left(x, u_{n}\right)-\frac{1}{2} D_{s} a_{i j}\left(x, u_{n}\right), M b_{i j}\left(x, v_{n}\right)-\frac{1}{2} D_{s} b_{i j}\left(x, v_{n}\right)$ are positive for $M$ large enough. By Fatou's lemma, the weak convergence of $\left\{\left(u_{n}, v_{n}\right)\right\}$ and the fact that $\mu_{n} \int_{\Omega}\left(\left|\nabla u_{n}\right|^{4}+\left|\nabla v_{n}\right|^{4}\right)$ is bounded, we have

$$
\begin{aligned}
0 \leq & \int_{\Omega} \sum_{i, j=1}^{N} a_{i j}(x, u) D_{i} u D_{j} \xi \exp (-M u)+\int_{\Omega} \sum_{i, j=1}^{N} b_{i j}(x, v) D_{i} v D_{j} \eta \exp (-M v) \\
& -\int_{\Omega} \sum_{i, j=1}^{N}\left(M a_{i j}(x, u)-\frac{1}{2} D_{s} a_{i j}(x, u)\right) D_{i} u D_{j} u \xi \exp (-M u) \\
& -\int_{\Omega} \sum_{i, j=1}^{N}\left(M b_{i j}(x, v)-\frac{1}{2} D_{s} b_{i j}(x, v)\right) D_{i} v D_{j} v \eta \exp (-M v) \\
& -\frac{2 \alpha}{\alpha+\beta} \int_{\Omega}|u|^{\alpha-2}|v|^{\beta} u \xi \exp (-M u)-\frac{2 \beta}{\alpha+\beta} \int_{\Omega}|u|^{\alpha}|v|^{\beta-2} v \eta \exp (-M v) \\
= & \int_{\Omega} \sum_{i, j=1}^{N} a_{i j}(x, u) D_{i} u D_{j}(\xi \exp (-M u))+\int_{\Omega} \sum_{i, j=1}^{N} b_{i j}(x, u) D_{i} v D_{j}(\eta \exp (-M v)) \\
& +\frac{1}{2} \int_{\Omega} \sum_{i, j=1}^{N} D_{s} a_{i j}(x, u) D_{i} u D_{j} u \xi \exp (-M u) \\
& +\frac{1}{2} \int_{\Omega} \sum_{i, j=1}^{N} D_{s} b_{i j}(x, v) D_{i} v D_{j} v \eta \exp (-M v) \\
& -\frac{2 \alpha}{\alpha+\beta} \int_{\Omega}|u|^{\alpha-2}|v|^{\beta} u \xi \exp (-M u)-\frac{2 \beta}{\alpha+\beta} \int_{\Omega}|u|^{\alpha}|v|^{\beta-2} v \eta \exp (-M v) .
\end{aligned}
$$


Let $(\chi, \omega) \geq(0,0),(\chi, \omega) \in C_{0}^{\infty}(\Omega) \times C_{0}^{\infty}(\Omega)$. We may choose $\xi=\chi \exp (M u), \eta=\omega \exp (M v)$ such that $(\xi, \eta) \in W_{0}^{1,4}(\Omega) \times W_{0}^{1,4}(\Omega),|\xi|_{L^{\infty}(\Omega)} \leq C$ and $|\eta|_{L^{\infty}(\Omega)} \leq C$. Then we obtain

$$
\begin{aligned}
& \int_{\Omega} \sum_{i, j=1}^{N} a_{i j}(x, u) D_{i} u D_{j} \chi+\int_{\Omega} \sum_{i, j=1}^{N} b_{i j}(x, v) D_{i} v D_{j} \omega \\
& +\frac{1}{2} \int_{\Omega} \sum_{i, j=1}^{N} D_{s} a_{i j}(x, u) D_{i} u D_{j} u \chi+\frac{1}{2} \int_{\Omega} \sum_{i, j=1}^{N} D_{s} b_{i j}(x, v) D_{i} v D_{j} v \omega \\
& -\frac{2 \alpha}{\alpha+\beta} \int_{\Omega}|u|^{\alpha-2}|v|^{\beta} u \chi-\frac{2 \beta}{\alpha+\beta} \int_{\Omega}|u|^{\alpha}|v|^{\beta-2} v \omega \geq 0
\end{aligned}
$$

for all $(\chi, \omega) \geq(0,0),(\chi, \omega) \in C_{0}^{\infty}(\Omega) \times C_{0}^{\infty}(\Omega)$.

Similarly, we may obtain an opposite inequality. Thus we have

$$
\begin{aligned}
& \int_{\Omega} \sum_{i, j=1}^{N} a_{i j}(x, u) D_{i} u D_{j} \chi+\int_{\Omega} \sum_{i, j=1}^{N} b_{i j}(x, v) D_{i} v D_{j} \omega \\
& +\frac{1}{2} \int_{\Omega} \sum_{i, j=1}^{N} D_{s} a_{i j}(x, u) D_{i} u D_{j} u \chi+\frac{1}{2} \int_{\Omega} \sum_{i, j=1}^{N} D_{s} b_{i j}(x, v) D_{i} v D_{j} v \omega \\
& -\frac{2 \alpha}{\alpha+\beta} \int_{\Omega}|u|^{\alpha-2}|v|^{\beta} u \chi-\frac{2 \beta}{\alpha+\beta} \int_{\Omega}|u|^{\alpha}|v|^{\beta-2} v \omega=0
\end{aligned}
$$

for all $(\chi, \omega) \in C_{0}^{\infty}(\Omega) \times C_{0}^{\infty}(\Omega)$. That is, $(u, v)$ is a critical point of $I_{0}$ and a solution for system (1.1). By doing approximations, we have $(u, v)$ in the place of $(\chi, \omega)$ of $(5.7)$

$$
\begin{aligned}
& \int_{\Omega} \sum_{i, j=1}^{N} a_{i j}(x, u) D_{i} u D_{j} u+\int_{\Omega} \sum_{i, j=1}^{N} b_{i j}(x, v) D_{i} v D_{j} v \\
& +\frac{1}{2} \int_{\Omega} \sum_{i, j=1}^{N} D_{s} a_{i j}(x, u) u D_{i} u D_{j} u \\
& +\frac{1}{2} \int_{\Omega} \sum_{i, j=1}^{N} D_{s} b_{i j}(x, v) v D_{i} v D_{j} v-2 \int_{\Omega}|u|^{\alpha}|v|^{\beta}=0 .
\end{aligned}
$$

Setting $(\varphi, \psi)=\left(u_{n}, v_{n}\right)$ in (5.1), we have

$$
\begin{aligned}
& \mu_{n} \int_{\Omega}\left(\left|\nabla u_{n}\right|^{4}+\left|\nabla v_{n}\right|^{4}\right)+\int_{\Omega} \sum_{i, j=1}^{N} a_{i j}\left(x, u_{n}\right) D_{i} u_{n} D_{j} u_{n} \\
& +\int_{\Omega} \sum_{i, j=1}^{N} b_{i j}\left(x, v_{n}\right) D_{i} v_{n} D_{j} v_{n}+\frac{1}{2} \int_{\Omega} \sum_{i, j=1}^{N} D_{s} a_{i j}\left(x, u_{n}\right) u_{n} D_{i} u_{n} D_{j} u_{n} \\
& \quad+\frac{1}{2} \int_{\Omega} \sum_{i, j=1}^{N} D_{s} b_{i j}\left(x, v_{n}\right) v_{n} D_{i} v_{n} D_{j} v_{n}-2 \int_{\Omega}\left|u_{n}\right|^{\alpha}\left|v_{n}\right|^{\beta}=0 .
\end{aligned}
$$


Using $\int_{\Omega}\left|u_{n}\right|^{\alpha}\left|v_{n}\right|^{\beta} \rightarrow \int_{\Omega}|u|^{\alpha}|v|^{\beta}$ as $n \rightarrow \infty$, (5.8), (5.9) and lower semi-continuity, we obtain

$$
\begin{aligned}
& \mu_{n} \int_{\Omega}\left(\left|\nabla u_{n}\right|^{4}+\left|\nabla v_{n}\right|^{4}\right) \rightarrow 0, \\
& \int_{\Omega} \sum_{i, j=1}^{N} a_{i j}\left(x, u_{n}\right) D_{i} u_{n} D_{j} u_{n} \rightarrow \int_{\Omega} \sum_{i, j=1}^{N} a_{i j}(x, u) D_{i} u D_{j} u, \\
& \int_{\Omega} \sum_{i, j=1}^{N} b_{i j}\left(x, v_{n}\right) D_{i} v_{n} D_{j} v_{n} \rightarrow \int_{\Omega} \sum_{i, j=1}^{N} b_{i j}(x, v) D_{i} v D_{j} v
\end{aligned}
$$

as $n \rightarrow \infty$.

In particular, we have

$$
\begin{aligned}
& u_{n} \rightarrow u, \quad v_{n} \rightarrow v \quad \text { in } W_{0}^{1,2}(\Omega), \\
& u_{n} \nabla u_{n} \rightarrow u \nabla u, \quad v_{n} \nabla v_{n} \rightarrow v \nabla v \quad \text { in } L^{2}(\Omega)
\end{aligned}
$$

and

$$
I_{\mu_{n}}^{\prime}\left(u_{n}, v_{n}\right) \rightarrow I_{0}^{\prime}(u, v)
$$

as $n \rightarrow \infty$. This completes the proof of Theorem 2.1.

Next, we apply the mountain pass theorem to obtain the existence of critical points of $I_{\mu}$. Set

$$
\begin{aligned}
\Sigma_{\rho}= & \left\{(u, v) \in W_{0}^{1,4}(\Omega) \times W_{0}^{1,4}(\Omega) \mid\right. \\
& \left.\int_{\Omega} \sum_{i, j=1}^{N} a_{i j}(x, u) D_{i} u D_{j} u+\int_{\Omega} \sum_{i, j=1}^{N} b_{i j}(x, v) D_{i} v D_{j} v \leq \rho^{2}\right\}
\end{aligned}
$$

for $\rho>0$.

Let us consider the functional

$$
\begin{aligned}
I_{\mu}^{+}(u, v)= & \frac{1}{4} \mu \int_{\Omega}\left(|\nabla u|^{4}+|\nabla v|^{4}\right)+\frac{1}{2} \int_{\Omega} \sum_{i, j=1}^{N} a_{i j}(x, u) D_{i} u D_{j} u \\
& +\frac{1}{2} \int_{\Omega} \sum_{i, j=1}^{N} b_{i j}(x, v) D_{i} v D_{j} v-\frac{2}{\alpha+\beta} \int_{\Omega}\left(u^{+}\right)^{\alpha}\left(v^{+}\right)^{\beta} .
\end{aligned}
$$

Here and in what follows, we denote $u^{+}=\max \{u, 0\}$. The functional $I_{\mu}$ satisfies $(P S)_{c}$ condition. Similarly, we may verify that $I_{\mu}^{+}$satisfies $(P S)_{c}$ condition. By the $\varepsilon$-Young inequality, for any $\varepsilon>0$, there exists $C_{\varepsilon}>0$ such that

$$
\left(u^{+}\right)^{\alpha}\left(v^{+}\right)^{\beta} \leq \varepsilon\left(u^{+}\right)^{\alpha+\beta}+C_{\varepsilon}\left(v^{+}\right)^{\alpha+\beta}
$$


and

$$
\begin{aligned}
& \int_{\Omega}|u|^{\alpha+\beta} \leq C\left(\int_{\Omega} u^{2}|\nabla u|^{2}\right)^{\frac{\alpha+\beta}{4}} \leq C\left(\int_{\Omega} \sum_{i, j=1}^{N} a_{i j}(x, u) D_{i} u D_{j} u\right)^{\frac{\alpha+\beta}{4}}, \\
& \int_{\Omega}|v|^{\alpha+\beta} \leq C\left(\int_{\Omega} v^{2}|\nabla v|^{2}\right)^{\frac{\alpha+\beta}{4}} \leq C\left(\int_{\Omega} \sum_{i, j=1}^{N} b_{i j}(x, v) D_{i} v D_{j} v\right)^{\frac{\alpha+\beta}{4}} .
\end{aligned}
$$

Then

$$
\begin{aligned}
& -\frac{2}{\alpha+\beta} \int_{\Omega}\left(u^{+}\right)^{\alpha}\left(v^{+}\right)^{\beta} \\
& \quad \geq-\frac{2}{\alpha+\beta} \varepsilon \int_{\Omega}\left(u^{+}\right)^{\alpha+\beta}-\frac{2}{\alpha+\beta} C_{\varepsilon} \int_{\Omega}\left(u^{+}\right)^{\alpha+\beta} \\
& \quad \geq-\frac{2 C}{\alpha+\beta} \varepsilon\left(\int_{\Omega} \sum_{i, j=1}^{N} a_{i j}(x, u) D_{i} u D_{j} u\right)^{\frac{\alpha+\beta}{4}}-\frac{2 C_{\varepsilon}}{\alpha+\beta}\left(\int_{\Omega} \sum_{i, j=1}^{N} b_{i j}(x, v) D_{i} v D_{j} v\right)^{\frac{\alpha+\beta}{4}} \\
& \quad \geq-\frac{2 C}{\alpha+\beta} \varepsilon \rho^{\frac{\alpha+\beta}{2}}-\frac{2 C_{\varepsilon}}{\alpha+\beta} \rho^{\frac{\alpha+\beta}{2}} \\
& \quad \geq-\frac{1}{\alpha+\beta} \rho^{2}
\end{aligned}
$$

for $\varepsilon, \rho$ small. Thus we have

$$
\begin{aligned}
I_{\mu}^{+}(u, v) & \geq \frac{1}{2} \int_{\Omega} \sum_{i, j=1}^{N} a_{i j}(x, u) D_{i} u D_{j} u+\frac{1}{2} \int_{\Omega} \sum_{i, j=1}^{N} b_{i j}(x, v) D_{i} v D_{j} v-\frac{2}{\alpha+\beta} \int_{\Omega}\left(u^{+}\right)^{\alpha}\left(v^{+}\right)^{\beta} \\
& \geq \frac{1}{2} \rho^{2}-\frac{1}{\alpha+\beta} \rho^{2}=\left(\frac{1}{2}-\frac{1}{\alpha+\beta}\right) \rho^{2}
\end{aligned}
$$

for $(u, v) \in \partial \Sigma_{\rho}$ and for $\rho>0$ small enough. Choose $(\varphi, \psi) \geq(0,0),(\chi, \omega) \in C_{0}^{\infty}(\Omega) \times$ $C_{0}^{\infty}(\Omega)$ and $T>0$. Define a path $(g, h):[0,1] \rightarrow W_{0}^{1,4}(\Omega) \times W_{0}^{1,4}(\Omega)$ by $(g(t), h(t))=$ $(t T \varphi, t T \psi)$. When $T$ is large enough, we have

$$
\begin{aligned}
& I_{\mu}^{+}(g(1), h(1))<0, \\
& \int_{\Omega} \sum_{i, j=1}^{N} a_{i j}(x, g(1)) D_{i} g(1) D_{j} g(1)+\int_{\Omega} \sum_{i, j=1}^{N} b_{i j}(x, h(1)) D_{i} h(1) D_{j} h(1)>\rho^{2}
\end{aligned}
$$

and

$$
\sup _{t \in[0,1]} I_{\mu}^{+}(g(t), h(t)) \leq m
$$

for some $m$ independent of $\mu \in(0,1]$.

Define

$$
c_{\mu}=\inf _{(g, h) \in \Gamma} \sup _{t \in[0,1]} I_{\mu}^{+}(g(t), h(t)),
$$


where

$$
\begin{aligned}
\Gamma= & \left\{(g, h) \in C\left([0,1], W_{0}^{1,4}(\Omega) \times W_{0}^{1,4}(\Omega)\right) \mid\right. \\
& (g(0), h(0))=(0,0),(g(1), h(1))=(T \varphi, T \psi)\} .
\end{aligned}
$$

From the mountain pass theorem we obtain that

$$
c_{\mu} \geq\left(\frac{1}{2}-\frac{1}{\alpha+\beta}\right) \rho^{2}
$$

is a critical value of $I_{\mu}^{+}$.

Let $\left(u_{\mu}, v_{\mu}\right)$ be a critical point corresponding to $c_{\mu}$. We have $\left(u_{\mu}, v_{\mu}\right) \geq(0,0)$. Thus $\left(u_{\mu}, v_{\mu}\right)$ is a positive critical point of $I_{\mu}$ by the strong maximum principle. In summary, we have the following.

Proposition 5.1 There exist positive constants $\rho$ and $m$ independent of $\mu$ such that $I_{\mu}$ has a positive critical point $\left(u_{\mu}, v_{\mu}\right)$ satisfying

$$
\left(\frac{1}{2}-\frac{1}{\alpha+\beta}\right) \rho^{2} \leq I_{\mu}\left(u_{\mu}, v_{\mu}\right) \leq m .
$$

Finally, we give the proof of Theorem 2.2 .

Proof of Theorem 2.2 For a positive solution of system (1.1), the proof follows from Proposition 5.1 and Theorem 2.1. A similar argument gives a negative solution of system (1.1). This completes the proof of Theorem 2.2.

\section{Competing interests}

The authors declare that they have no competing interests.

\section{Authors' contributions}

All the authors were involved in carrying out this study. All authors read and approved the final manuscript.

\section{Author details}

${ }^{1}$ College of Mathematics and Computer Science, Northwest University for Nationalities, Lanzhou, 730124, P.R. China.

${ }^{2}$ College of Mathematics and Statistics, Northwest Normal University, Lanzhou, 730070, P.R. China. ${ }^{3}$ School of Mathematical Sciences, Beijing Normal University, Beijing, 100875, P.R. China.

\section{Acknowledgements}

This paper was finished while the first author was a visiting fellow at the School of Mathematical Sciences of Beijing Normal University, and the first author would like to express her gratitude for their hospitality during her visit. This work is supported by the National Science Foundation of China (11061031), Fundamental Research Funds for the Central

Universities (31920130004) and Fundamental Research Funds for the Gansu University.

\section{Received: 28 May 2013 Accepted: 28 August 2013 Published: 07 Nov 2013}

\section{References}

1. Poppenberg, M, Schmitt, K, Wang, Z: On the existence of soliton solutions to quasilinear Schrödinger equations. Calc. Var. Partial Differ. Equ. 14, 329-344 (2002)

2. Liu, J, Wang, Y, Wang, Z: Soliton solutions for quasilinear Schrödinger equation, II. J. Differ. Equ. 187, 473-493 (2003)

3. Colin, M, Jeanjean, L: Solutions for a quasilinear Schrödinger equation: a dual approach. Nonlinear Anal. 56, 213-226 (2004)

4. Berestycki, H, Lions, PL: Nonlinear scalar field equations, I. Arch. Ration. Mech. Anal. 82, 313-346 (1983)

5. Liu, J, Wang, Y, Wang, Z: Solutions for quasilinear Schrödinger equations via the Nehari method. Commun. Partial Differ. Equ. 29, 879-901 (2004)

6. Lions, PL: The concentration-compactness principle in the calculus of variations. The locally compact case. Part I. Ann. Inst. Henri Poincaré, Anal. Non Linéaire 1, 109-145 (1984) 
7. Guo, Y, Tang, Z: Ground state solutions for the quasilinear Schrödinger systems. J. Math. Anal. Appl. 389, 322-339 (2012)

8. Guo, Y, Tang, Z: Ground state solutions for the quasilinear Schrödinger equation. Nonlinear Anal. 75, 3235-3248 (2012)

9. Liu, X, Liu, J, Wang, Z: Quasilinear elliptic equations via perturbation method. Proc. Am. Math. Soc. 141, 253-263 (2013)

10. Bartsch, T, Wang, Z: Multiple positive solutions for a nonlinear Schrödinger equation. Z. Angew. Math. Phys. 51 366-384 (2000)

11. Ambrosetti, A, Badiale, M, Cingolani, S: Semiclassical states of nonlinear Schrödinger equations. Arch. Ration. Mech. Anal. 140, 285-300 (1997)

12. Ambrosetti, A, Malchiodi, A, Secchi, S: Multiplicity results for some nonlinear Schrödinger equations with potentials. Arch. Ration. Mech. Anal. 159, 253-271 (2001)

13. Byeon, J, Wang, Z: Standing waves with a critical frequency for nonlinear Schrödinger equations, II. Calc. Var. Partial Differ. Equ. 18, 207-219 (2003)

14. Cingolani, S, Lazzo, M: Multiple positive solutions to nonlinear Schrödinger equations with competing potential functions. J. Differ. Equ. 160, 118-138 (2000)

15. Cingolani, S, Nolasco, M: Multi-peaks periodic semiclassical states for a class of nonlinear Schrödinger equations. Proc R. Soc. Edinb. 128, 1249-1260 (1998)

16. Del Pino, M, Felmer, P: Semi-classical states for nonlinear Schrödinger equations. Ann. Inst. Henri Poincaré 15, 127-149 (1998)

17. Del Pino, M, Felmer, P: Multi-peak bound states for nonlinear Schrödinger equations. J. Funct. Anal. 149, 245-265 (1997)

18. Floer, A, Weinstein, A: Nonspreading wave packets for the cubic Schrödinger equation with a bounded potential. J. Funct. Anal. 69, 397-408 (1986)

19. Oh, YG: On positive multi-bump bound states of nonlinear Schrödinger equations under multiple well potential. Commun. Math. Phys. 131, 223-253 (1990)

20. Oh, YG: Existence of semiclassical bound states of nonlinear Schrödinger equations with potentials of class $(V)_{a}$. Commun. Partial Differ. Equ. 13, 1499-1519 (1988)

21. Liu, J, Wang, Z: Soliton solutions for quasilinear Schrödinger equations, I. Proc. Am. Math. Soc. 131, 441-448 (2003)

22. Canino, A, Degiovanni, M: Nonsmooth critical point theory and quasilinear elliptic equations. In: Topological Methods in Differential Equations and Inclusions (Montreal, PQ, 1994). NATO Adv. Sci. Inst. Ser. C Math. Phys. Sci., vol. 472, pp. 1-50 (1995)

23. Liu, J, Wang, Z: Bifurcations for quasilinear Schrödinger equations, II. Commun. Contemp. Math. 10, $723-743$ (2008)

10.1186/1687-2770-2013-219

Cite this article as: Jiao et al.: Existence of solutions for a general quasilinear elliptic system via perturbation method. Boundary Value Problems 2013, 2013:219

\section{Submit your manuscript to a SpringerOpen ${ }^{\circledR}$ journal and benefit from:}

- Convenient online submission

Rigorous peer review

- Immediate publication on acceptance

- Open access: articles freely available online

- High visibility within the field

- Retaining the copyright to your article 